\title{
Is spacetime hole-free?
}

\author{
John Byron Manchak
}

Received: 24 September 2008 / Accepted: 27 November 2008 / Published online: 14 December 2008 (C) The Author(s) 2008. This article is published with open access at Springerlink.com

\begin{abstract}
Here, we examine hole-freeness - a condition sometimes imposed to rule out seemingly artificial spacetimes. We show that under existing definitions (and contrary to claims made in the literature) there exist inextendible, globally hyperbolic spacetimes which fail to be hole-free. We then propose an updated formulation of the condition which enables us to show the intended result. We conclude with a few general remarks on the strength of the definition and then formulate a precise question which may be interpreted as: Are all physically reasonable spacetimes hole-free?
\end{abstract}

Keywords Hole-freeness · Determinism - Global structure $\cdot$ Singularities

\section{Introduction}

Hole-freeness is a condition sometimes imposed to rule out seemingly artificial spacetimes. The idea, roughly, is that spacetime should be such that the Cauchy development of any spacelike surface is "as large as it can be". The condition is of interest because, without it, it seems that a failure of determinism in general relativity threatens even before one considers more interesting questions of global structure such as the cosmic censorship hypothesis. ${ }^{1}$ The first precise formulation of hole-freeness was due to Geroch [8]: ${ }^{2}$

\footnotetext{
1 See Earman [6, pp. 97-98].

2 In addition to [8], precise definitions of hole-freeness can be found in Clarke [1-3] and Earman [5,6]. For other discussions of hole-freeness, see Clarke and Schmidt [4] and Ellis and Schmidt [7].
}

J. B. Manchak ( $\bowtie)$

Logic and Philosophy of Science, UC, Irvine, USA

e-mail: jmanchak@uci.edu 
Definition A spacetime $\left(M, g_{a b}\right)$ is said to be hole-free if, for any spacelike surface $S \subset M$, and for any metric preserving embedding $\theta: D(S) \rightarrow M^{\prime}$ into another spacetime $\left(M^{\prime}, g_{a b}^{\prime}\right), \theta(D(S))=D(\theta(S)) .^{3}$

To see the definition at work, one can verify that Minkowski spacetime $\left(\mathbb{R}^{4}, \eta_{a b}\right)$ is hole-free. On the other hand, consider the spacetime $\left(\mathbb{R}^{4}-\{p\}, \eta_{a b}\right)$ where $p$ is any point in $\mathbb{R}^{4}$. This spacetime is Minkowski spacetime with a point removed and clearly it is not hole-free. One naturally wonders about the strength of the definition. Geroch [8] noted that this formulation may be too weak-Minkowski spacetime to the past of a null plane counts as hole-free. Similarly, the definition may also be too strong. It has been noted by Clarke [1], for example, that there are spacetimes representing spherically symmetric radiating stars which are counted as not hole-free. ${ }^{4}$ However, it was suggested by Ellis and Schmidt [7] that every inextendible, globally hyperbolic spacetime is hole-free (a proof of this claim was later given in Clarke [3]). Thus, it seemed that at least a large class of physically reasonable spacetimes must be hole-free. However, we will show here that contrary to these claims, a spacetime may indeed be inextendible, globally hyperbolic, and not hole-free. ${ }^{5}$

Proposition 1 There exists an inextendible, globally hyperbolic spacetime which is not hole-free.

Proof Let $\left(\mathbb{R}^{2}, \eta_{a b}\right)$ be (two-dimensional) Minkowski spacetime in standard $(t, x)$ coordinates. Now let $p \in \mathbb{R}^{2}$ be the origin point $(0,0)$ and consider the set $J^{-}(p) \subset$ $\mathbb{R}^{2}$. Call $K$ the set $J^{-}(p)-\{p\}$. Finally, let $M$ be the set $\{(t, x): t<0\}$. Consider the (smooth, strictly positive) scalar function $\Omega: M \rightarrow \mathbb{R}$ such that (i) $\Omega(t, x)=1$ for all $(t, x) \in K$ and (ii) $\Omega_{\mid M-K}(t, x) \rightarrow 0$ as $t \rightarrow 0$. Let $g_{a b}=\Omega^{2} \eta_{a b}$ and consider the spacetime $\left(M, g_{a b}\right)$. Clearly, such a spacetime cannot be extended. Consider the (achronal) set of points $\Sigma=\{(t, x) \in M: t=-1\}$. Because $\left(M, g_{a b}\right)$ is conformally flat, it can be easily verified that $D(\Sigma)=M$. So, $\Sigma$ is a Cauchy surface and therefore $\left(M, g_{a b}\right)$ is globally hyperbolic.

Finally, we show that $\left(M, g_{a b}\right)$ is not hole-free. Consider the achronal set $S=\Sigma \cap K$ in $M$. Clearly, $D(S) \subset K$ and thus, for each point in $D(S)$, we have $g_{a b}=\eta_{a b}$. Let $\theta$ : $D(S) \rightarrow \mathbb{R}^{2}$ be an isometric embedding of $D(S)$ into Minkowski spacetime $\left(\mathbb{R}^{2}, \eta_{a b}\right)$ such that $\theta=i d$. We claim that $\theta(D(S)) \neq D(\theta(S))$ and hence, that $\left(M, g_{a b}\right)$ is not hole-free. One can see this by noting that the origin point $p=(0,0) \in \mathbb{R}^{2}$ is such that $p \in D(\theta(S))$ but $p \notin \theta(D(S)))$. Thus, there exists an inextendible spacetime which is globally hyperbolic and not hole-free.

This result is surprising not only because it contradicts assertions made in the literature, but because it seems to contradict our intuitions concerning the intended relationship between inextendibility, global hyperbolicity, and hole-freeness. To help

\footnotetext{
${ }^{3}$ Here $D(S)$ is the domain of dependence of $S$. For the sake of continuity later, we define $D(S)$ as does Wald [12].

4 See Steinmüller, King, and Lasota [11] for details.

5 Although Clarke's [3] proof utilized a variant definition of hole-freeness, it can be easily verified that the counterexample presented below applies just as well to this alternate formulation.
} 
emphasize the point, consider that, for intuitive reasons, inextendible spacetimes are sometimes called "maximal", globally hyperbolic spacetimes are sometimes called "deterministic", and hole-free spacetimes are sometimes called "determinism maximal". 6 With this seemingly benign translation available, we can restate the theorem above as follows: there are deterministic, maximal spacetimes which are not determinism maximal!

Of course, there is no serious problem here. We simply must find an alternate (weaker) formulation of hole-freeness which seems to capture the spirit of the definition and, at the same same, allows one to prove the intended theorem. Such an alternate definition has been provided by Geroch. ${ }^{7}$ We present the updated formulation here in two stages.

Definition Let $\left(M, g_{a b}\right)$ be a globally hyperbolic spacetime. Call an extension $\left(M^{\prime}, g_{a b}^{\prime}\right)$ of $\left(M, g_{a b}\right)$ effective if, for some Cauchy surface $S$ in $\left(M, g_{a b}\right)$, the set $\operatorname{int}[D(S)]$ in the extension $\left(M^{\prime}, g_{a b}^{\prime}\right)$ is such that (i) $S$ remains achronal and (ii) $M \subsetneq \operatorname{int}[D(S)]$.

Definition A spacetime $\left(M, g_{a b}\right)$ is hole-free* if, for every set $K \subseteq M$ such that $\left(K, g_{a b \mid K}\right)$ is a globally hyperbolic spacetime, if there exists an effective extension for $\left(K, g_{a b \mid K}\right)$, then $\left(M, g_{a b}\right)$ is an effective extension for $\left(K, g_{a b \mid K}\right)$.

Clearly, the spirit of hole-freeness is captured in this version of the definition. That it is weaker than the traditional formulation is given by the following proposition.

Proposition 2 If a spacetime $\left(M, g_{a b}\right)$ is hole-free, then it is also hole-free*.

Proof Assume a spacetime $\left(M, g_{a b}\right)$ is hole-free. Let $K \subseteq M$ be such that $\left(K, g_{a b \mid K}\right)$ is a globally hyperbolic spacetime. Assume that there is an effective extension $\left(M^{\prime}, g_{a b}^{\prime}\right)$ for $\left(K, g_{a b \mid K}\right)$ and that $\left(M, g_{a b}\right)$ is not such an effective extension (assume it is not hole-free*). We show a contradiction. Let $S$ be a Cauchy surface in $\left(K, g_{a b \mid K}\right)$. So, for some isometric embedding $\theta: K \rightarrow M^{\prime}$, we have that $\theta(K) \subsetneq$ $\operatorname{int}[D(\theta(S))]$. Because $\theta(K)$ is open, int $[\theta(K)]=\theta(K)$. So, this implies that $\operatorname{int}[\theta(K)] \subsetneq \operatorname{int}[D(\theta(S))]$. But, because $\left(M, g_{a b}\right)$ is hole-free, then $\theta(D(S))=$ $\theta(K)=D(\theta(S))$. So, int $[\theta(K)]=\operatorname{int}[D(\theta(S))]$ and we have our contradiction.

Our weakened formulation of hole-freeness allows us to prove the intuitive result discussed above. (Taken together, the result below along with proposition 1 show that hole-freeness and hole-freeness* are not equivalent definitions.)

Proposition 3 Every inextendible, globally hyperbolic spacetime is hole-free*.

Proof Let $\left(M, g_{a b}\right)$ be any globally hyperbolic spacetime which fails to be hole-free. We show that it is extendible. So there exists a $K \subseteq M$ such that $\left(K, g_{a b \mid K}\right)$ is a globally hyperbolic spacetime and (i) there exists an effective extension $\left(N, g_{a b}^{\prime}\right)$ of $\left(K, g_{a b \mid K}\right)$ and (ii) $\left(M, g_{a b}\right)$ is not an effective extension for $\left(K, g_{a b \mid K}\right)$.

\footnotetext{
6 See Earman [6, p. 32, 44, 98].

7 Private communication.
} 
Let $K^{\prime} \subsetneq N$ be such that there exists an isometry $\theta: K \rightarrow K^{\prime}$. Let $S$ be a Cauchy surface in $\left(K, g_{a b \mid K}\right)$. By definition, $K^{\prime} \subsetneq \operatorname{int}[D(\theta(S))]$. Let $N^{\prime}=\operatorname{int}[D(\theta(S))]$. Now let $M^{\prime}=\left(M \cup N^{\prime}\right) / \theta$ (i.e. the quotient of the disjoint union of $M$ and $N^{\prime}$ under the equivalence relation $x \sim y \equiv x=y$ or $\theta(x)=y$ or $\theta(y)=x)$.

The remainder of the proof follows that of proposition 6.5.1 given by Clarke [3].

We conclude with a few thoughts on the strength of hole-freeness. More specifically, we wonder if the following statement is true in some sense: All physically reasonable spacetimes are hole-free (call this statement the "hole-free hypothesis"). The (strong) cosmic censorship hypothesis states that every physically reasonable spacetime is globally hyperbolic. ${ }^{8}$ So, if the cosmic censorship hypothesis is true, one must only assume that spacetime is inextendible to show, via the theorem above, that the hole-free hypothesis is true. However, at present, the cosmic censorship hypothesis is still very much an open question. Accordingly, we would like to consider the status of the hole-free hypothesis in the event that the cosmic censorship hypothesis is false. First, we must arrive at a precise formulation of the statement.

A natural starting place for such a task is to wonder about the prospects of weakening the global hyperbolicity assumption in the theorem above. For example, it would be interesting to show that every inextendible, stably causal spacetime satisfying some relatively weak auxiliary assumptions (such as the energy conditions, etc.) must be hole-free. However, a hole-free hypothesis of this kind seems doomed to failure. We know, for example, that there are spacetimes which are inextendible, stably causal, are locally isometric to Minkowski spacetime, and also fail to be hole-free. ${ }^{9}$

A more promising formulation of the hole-free hypothesis would be to mimic the evolutionary approach often used in framing precise statements of the cosmic censorship hypothesis. ${ }^{10}$ Here is one such formulation.

Conjecture If the initial data set $\left(\Sigma, h_{a b}, K_{a b}\right)$ constitutes a counterexample to the cosmic censorship hypothesis found in Wald [12, p. 305], then there exists some extension to the maximal Cauchy development of $\left(\Sigma, h_{a b}, K_{a b}\right)$ which is inextendible, hole-free, and satisfies the dominant energy condition.

The idea here is that, if it turns out that the maximal Cauchy development $\left(M, g_{a b}\right)$ of some initial data set $\left(\Sigma, h_{a b}, K_{a b}\right)$ is both physically reasonable and extendible, then one would expect that there would be some extension to $\left(M, g_{a b}\right)$ which is physically reasonable. Notice that the structure of this conjecture is such that it is implied by the cosmic censorship hypothesis. ${ }^{11}$

It might seem that this formulation of the hole-free hypothesis must certainly be true. However, Clarke [1] has shown that not every hole-free spacetime has a hole-free inextendible extension. Referring to Clarke's work, Earman [5, p. 184] speculates: "Perhaps there are plausible regularity conditions on initial data which will rule out

\footnotetext{
8 See Wald [12, p. 304-305].

9 See Hawking and Ellis [10, p. 58-59] for an example.

10 See Geroch and Horowitz [9] and Wald [12] for details.

11 If the cosmic censorship hypothesis is true, then the hole-free hypothesis is vacuously true.
} 
such latent holes but I know of no specific results on this point." Our conjecture above may be interpreted as an attempt to formalize Earman's intuitions.

It has been argued that one can have no disposition to the condition of hole-freeness except to assume that it holds by fiat. ${ }^{12}$ But, here we have outlined another disposition. One may have an open perspective on the matter and attempt to show, from more basic assumptions, that hole-freeness is a reasonable condition to be placed on spacetime.

Acknowledgments I wish to thank John Earman and Robert Geroch for helpful discussions on this topic.

Open Access This article is distributed under the terms of the Creative Commons Attribution Noncommercial License which permits any noncommercial use, distribution, and reproduction in any medium, provided the original author(s) and source are credited.

\section{References}

1. Clarke, C.J.S.: Space-time singularities. Commun. Math. Phys. 49, 17-23 (1976)

2. Clarke, C.J.S.: Singularities: global and local Aspects. In: Bergmann, P.G., De Sabbata, V. (eds.) Topological Properties and Global Structure of Space-Time, pp. 61-72. Plenum Press, New York (1986)

3. Clarke, C.J.S.: The Analysis of Space-Time Singularities. Cambridge University Press, Cambridge (1993)

4. Clarke, C.J.S., Schmidt, B.: Singularities: The State of the Art. Gen. Relativ. Gravit. 8, 129-137 (1977)

5. Earman, J.: A Primer on Determinism. D. Reidel, Dordrecht (1986)

6. Earman, J.: Bangs, Crunches, Whimpers, and Shrieks. Oxford University Press, Oxford (1995)

7. Ellis, G.F.R., Schmidt, B.: Singular space-times. Gen Relativ Gravit 8, 915-953 (1977)

8. Geroch, R.: Prediction in general relativity. In: Earman, J., Glymour, C., Statchel, J. (eds.) Foundations of Space-Time Theories, Minnesota Studies in the Philosophy of Science, vol. VIII, pp. 81-93. University of Minnesota Press, Minneapolis (1977)

9. Geroch, R., Horowitz, G.: Global Structure of Spacetimes. In: Hawking, S., Isreal, W. (eds.) General Relativity: An Einstein Centenary Survey, pp. 212-293. Cambridge University Press, Cambridge (1979)

10. Hawking, S., Ellis, G.F.R.: The Large Scale Structure of Space-Time. Cambridge University Press, Cambridge (1973)

11. Steinmüller, B., King, A., Lasota, J.: Radiating bodies and naked singularites. Phys. Lett. A 51, 191 (1975)

12. Wald, R.: General Relativity. University of Chicago Press, Chicago (1984)

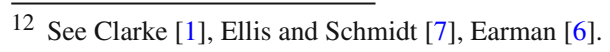

